\title{
Mandible and skull segmentation in cone beam computed tomography using super-voxels and graph clustering
}

\author{
Oscar Cuadros Linares ${ }^{1}(\mathbb{D}) \cdot$ Jonas Bianchi ${ }^{2} \cdot$ Dirceu Raveli $^{2} \cdot$ João Batista Neto $^{1} \cdot$ Bernd Hamann $^{3}$
}

๑) Springer-Verlag GmbH Germany, part of Springer Nature 2018

\begin{abstract}
Cone beam computed tomography $(\mathrm{CBCT})$ is a medical imaging technique employed for diagnosis and treatment of patients with cranio-maxillofacial deformities. CBCT 3D reconstruction and segmentation of bones such as mandible or maxilla are essential procedures in surgical and orthodontic treatments. However, CBCT image processing may be impaired by features such as low contrast, inhomogeneity, noise and artifacts. Besides, values assigned to voxels are relative Hounsfield units unlike traditional computed tomography (CT). Such drawbacks render CBCT segmentation a difficult and time-consuming task, usually performed manually with tools designed for medical image processing. We present an interactive two-stage method for the segmentation of CBCT: (i) we first perform an automatic segmentation of bone structures with super-voxels, allowing a compact graph representation of the 3D data; (ii) next, a user-placed seed process guides a graph partitioning algorithm, splitting the extracted bones into mandible and skull. We have evaluated our segmentation method in three different scenarios and compared the results with ground truth data of the mandible and the skull. Results show that our method produces accurate segmentation and is robust to changes in parameters. We also compared our method with two similar segmentation strategy and showed that it produces more accurate segmentation. Finally, we evaluated our method for CT data of patients with deformed or missing bones and the segmentation was accurate for all data. The segmentation of a typical CBCT takes in average 5 min, which is faster than most techniques currently available.
\end{abstract}

Keywords Cone beam computed tomography $\cdot$ Graph clustering $\cdot$ Super-voxels $\cdot$ Bone segmentation $\cdot$ Mandible $\cdot$ Skull

\section{Introduction}

Cone beam computed tomography (CBCT) is a dentomaxillofacial imaging technique developed by Mozzo et al. [23]. In the last years, it has become popular among dentists and researchers in dental sciences [22]. CBCT is employed in the diagnosis and treatment planning of bones such as dental and maxillofacial structures [23]. Differently from the traditional computed tomography $(\mathrm{CT})$, which uses a helical fan-beam technique, CBCT uses a cone beam

\section{Oscar Cuadros Linares}

ocuadros@icmc.usp.br

1 Instituto de Ciências Matemáticas e de Computação (ICMC), University of São Paulo (USP), São Carlos, SP 13566-590, Brazil

2 Faculdade de Odontologia (FOAR), São Paulo State University (UNESP), Araraquara, SP 14801-385, Brazil

3 Department of Computer Science, University of California, Davis, CA 95616, USA instead and produces images whose quality is adequate for diagnostic purposes. Besides, its radiation is approximately 98\% lower and also the scanning time is shorter when compared with fan-beam CT systems [23,29]. However, CBCT has some drawbacks such as artifacts caused by beam hardening, scattered radiation, low-contrast resolution, inhomogeneity, limited range of the X-ray area detectors and high resolution. Moreover, values assigned to voxels are relative Hounsfield units (HUs) [28] and cannot be precisely used as a threshold value to identify bone areas, as usually done in traditional CT [22]. These drawbacks have an impact not only on image quality but also on the outcome of image processing tasks [20].

CBCT is routinely used to study skull bones changes that occur over time, following orthodontic treatments. For example, the study of location and magnitude of facial asymmetry, congenital deformities, face deformities of mandibular joins, cranio-maxillofacial deformities and plan surgical correction $[6,31,35]$. Due to the complex nature of human skull, such treatments require extensive planning [6]. Hence, CT 3D 
reconstruction techniques and segmentation of skull bones, such as mandible or maxilla, are essential procedures for planning, performing and following-up orthodontic treatments [31].

Commonly, the segmentation of a CBCT data is "manually" performed and combines several computer tools specifically designed for medical image processing (i.e., ITK-Snap [37], 3D Slicer [12], Dolphin Imaging [30]). Attaining accurate $3 \mathrm{D}$ segmentation requires extensive training as it demands both knowledge about the complex anatomy of human skull and skills to properly operate medical image processing tools. Usually, such tools provide thresholding, level set and region-growing approaches which require intensive user interaction. Moreover, they are also very sensitive to artifacts, noise, low contrast and inhomogeneity (typical problems of CBCTs [33]). Nevertheless, these tools are popular among orthodontists despite the painstaking process involved: need to manually guide the entire segmentation process and the excessive time spent to generate acceptable results.

Other approaches to CT bone segmentation can be found in the literature, ranging from thresholding, region growing, deformable models, level set, graph cut, random forest classifiers, statistical shape models [32] and statistical atlases $[33,34,39]$. Most of them are ad hoc applications which handle one type of bone only: segmentation of either mandible or maxilla $[4,6,11,20,31]$.

Atlas-based methods aim to segment one category of object and can generate precise segmentation. However, they are very sensitive to anatomical variations, bone growth, for instance. Although such limitations may be circumvented by multiple atlases, the computational efficiency is sacrificed, especially when non-rigid registration is needed [39]. Besides, building atlases requires multiple training volumes, which can be hard to obtain $[7,33,34]$.

Graph-based methods are also widely used in medical image segmentation. In general, these methods build a graph, in which each pixel represents a vertex in the graph. The graph is then divided into clusters of vertices, which correspond to objects of interest in the input image [26]. Finding the best partition of a graph is an NP-hard problem, although optimal approximations may be obtained. Usually, such approximations divide a graph into clusters by minimizing an energy function [16]. Even though graph-based methods generate accurate segmentation, their major limitation is related to graph cardinality (number of vertices and number of edges): an image with $N$ pixels generates a graph whose adjacency matrix has $N^{2}$ elements, whereas a 3D image can easily yield a graph with millions of vertices and an even larger number of edges. Generally, graph cut approaches segment images automatically, i.e., no user interaction is needed during the process. There are, however, other graph-based methods in which users can interactively guide the segmen- tation process by manually placing seeds (labels) over objects of interest [36].

In this paper, we propose an interactive method to aid specialists in segmenting CBCT (Fig. 1). This includes the mandible and the skull altogether. It consists of a fully automatic pre-segmentation of the bony structure, followed by an interactive, user-driven segmentation, in which the aforementioned bones are accurately segmented. Given CBCT high resolution, its ability to exhibit very complex anatomical structures and the inherent existence of artifacts and low contrast, we highlight four features of our proposal:

1. Bone enhancement based on sheetness score [11] to enhance intensity of thin bones, such as maxilla.

2. Threshold-based bony structure pre-segmentation to separate bones from soft tissue, air and image background.

3. Super-voxels to reduce the excessive number of voxels of CBCT 3D data, leading to shorter processing time.

4. Interactive bone segmentation based on graph clustering.

The major contribution of this paper is the interactive segmentation of the mandible and the skull in a unified approach. Other important contributions are:

- Selection of bone of interest with simple brush strokes performed on any plane (sagittal, coronal and axial plane), tolerant to inaccuracies in positioning.

- Efficient segmentation method requiring about $5 \mathrm{~min}$ processing time to segment a typical CBCT image of resolution $400 \times 400 \times 554$.

This manuscript is organized as follows. Section 2 introduces the main concepts that underpin our method. In Sect. 3, we present our methodology. Section 4 describes the experiments and results. Conclusions and future work are given in Sect. 5 .

\section{Related concepts}

Our framework combines algorithms for bone enhancement in CT data, a super-voxel method and a seed-based graph cut approach to segment the bones of interest. We provide an overview of these concepts that are the foundation of our integrative method. Let $\Omega$ be a CBCT image and $\Omega_{i}$ the HU value of voxel $i \in n$, with $n$ being the number of voxels in $\Omega$.

\subsection{Sheetness score}

Low contrast in CBCT data is one of the major problems to be tackled for generating acceptable skull models from the data. In general, significant user input and steering are required to extract bone structure accurately [6]. Bone regions mostly affected by these problems are those containing thin bones, 
Fig. 1 Examples of our input CBCT datasets. Typical CBCT datasets have a resolution of $400 \times 400 \times 554$ (male and female patients), with an isotropic voxel size of $0.4 \mathrm{~mm}$, acquired with an i-Cat scanner. Upper row: dataset 1; lower row: dataset 2. From left to right: 3D rendering, sagittal, coronal and axial slices, respectively. Low-contrast and sheetlike bones are difficult to segment and are pointed out by a yellow arrow. a 3D view. b Condyle: low contrast. c Mandible ramus. d Mandible. e 3D view. $\mathbf{f}$ Palatine and maxilla. g Posterior view of Mandible and maxilla. $\mathbf{h}$ Sinus anterior wall

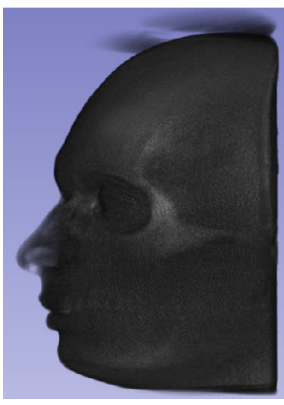

(a)

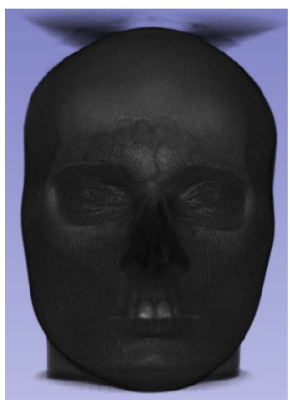

(e)

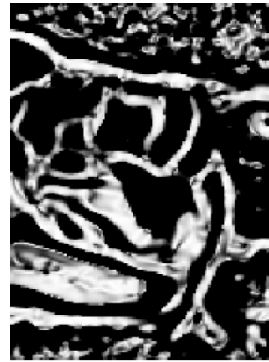

(c)

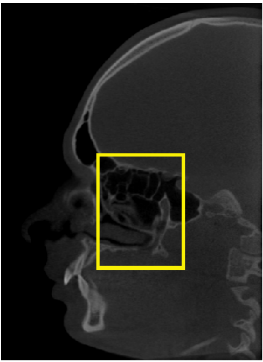

(a)

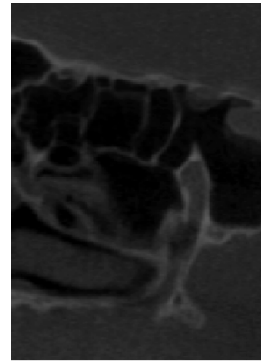

(b)

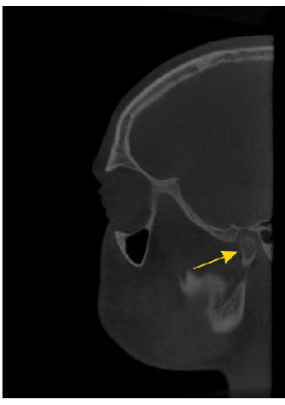

(b)

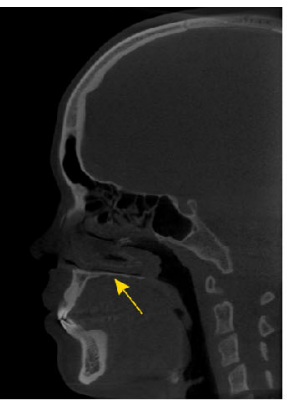

(f)

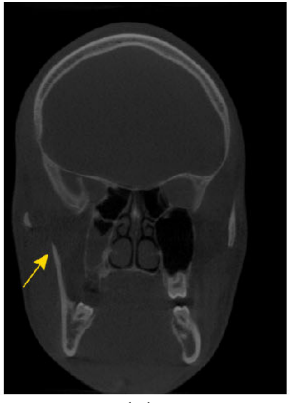

(c)

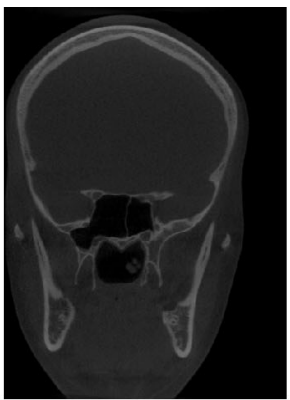

(g)

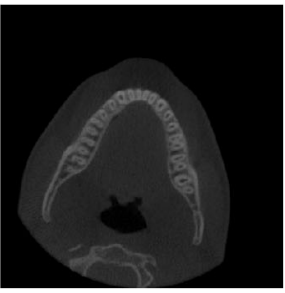

(d)

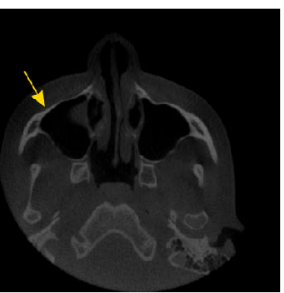

(h)
Fig. 2 Thin bones in CBTC data are hard to extract due to contrast problem. Results obtained with the sheetness score are shown in (c), successfully enhancing bones in the data, especially thin ones. a Sagittal slice with region of interest (yellow). b Close-up view of region of interest shown in (a). $\mathbf{c}$ Rendering of sheetness score of region of interest

e.g., sinus, palatine and maxilla (Fig. 2a). Traditional thresholding and region-growing methods fail to extract such bones accurately, leading to results with missing parts and "broken" contours, or even "leaks" into surrounding soft tissues [17].

The sheetness score enhances bony structures by an eigenvalue decomposition of the Hessian matrix. The information extracted from the Hessian matrix is commonly used to highlight curvilinear structures, e.g., vessels or bronchi in 3D medical images [15]. Because the eigenvalue decomposition measures the maximal change of the gradient vector of intensity in a local neighborhood, it can also be used as a means to identify tubelike, sheetlike and blob-like structures. Given a CBCT image $\Omega$, for each voxel $\Omega_{i}$ the sheetness score $S_{i}$ is defined by the eigenvalues $\left|\lambda_{1}\right| \leq\left|\lambda_{2}\right| \leq\left|\lambda_{3}\right|$ of the local Hessian matrix as follows:

$$
\begin{aligned}
S_{i}(\sigma)= & \max _{\sigma \in \Sigma}\left\{\exp \left[\frac{-R_{\text {sheet }}^{2}}{2 \alpha^{2}}\right]\right. \\
& \left.\times\left(1-\exp \left[\frac{-R_{\text {tube }}^{2}}{2 \beta^{2}}\right]\right)\left(1-\exp \left[\frac{-R_{\text {noise }}^{2}}{2 \gamma^{2}}\right]\right)\right\},
\end{aligned}
$$

where $R_{\text {sheet }}=\left|\lambda_{2}\right| /\left|\lambda_{3}\right|, R_{\text {tube }}=\left|\left(2\left|\lambda_{3}\right|-\left|\lambda_{2}\right|-\left|\lambda_{1}\right|\right)\right| /\left|\lambda_{3}\right|$ and $R_{\text {noise }}=\sqrt{\lambda_{1}^{2}+\lambda_{2}^{2}+\lambda_{3}^{2}}$. The values of $\alpha, \beta$ and $\gamma$ are set to $0.5,0.5$ and half the maximal value of the Frobenius norm $\left(R_{\text {noise }}\right)$, respectively. And $\Sigma$ is a set with values in the range $0.5 \leq \sigma \leq 3.0$ [11] used as parameters to compute the local Hessian matrix bt means of convolution with the second and cross-derivatives of a recursive Gaussian filter [21].

Equation 1 computes the Hessian matrix for all values of $\Sigma$ (a multi-scale process) and selects the maximum value in order to find structures of distinct thicknesses (vessels, for instance). In practice, this operation becomes computationally expensive as it is performed at every voxel in $\Omega$, for each element in $\Sigma$. Since our goal is to enhance thin structures only, we can drop the multi-scale process and compute the sheetness score for a single $\sigma$ value. The simplified Eq. 1 is given as follows:

$$
\begin{aligned}
S_{i}(\sigma)= & \exp \left[\frac{-R_{\text {sheet }}^{2}}{2 \alpha^{2}}\right] \\
& \times\left(1-\exp \left[\frac{-R_{\text {tube }}^{2}}{2 \beta^{2}}\right]\right)\left(1-\exp \left[\frac{-R_{\text {noise }}^{2}}{2 \gamma^{2}}\right]\right) .
\end{aligned}
$$




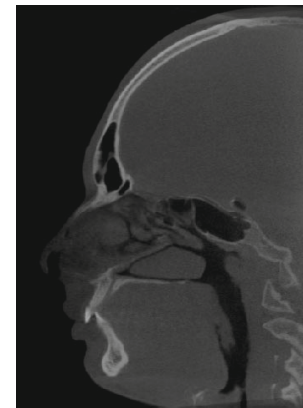

(a)

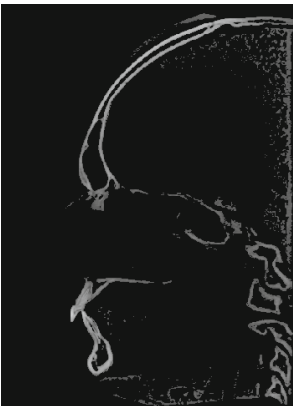

(b)
Fig. 3 Entire bone structure, including thin bones, extracted with the proposed threshold-based filter. a Input: sagittal CBCT slice. b Bone structure

However, parameter $\sigma$ must be given. In Sect. 4 , we give more details about parameter optimization and propose parameter values which guarantee optimal results. The sheetness score can be interpreted as the probability $S_{i}$ of a voxel belonging to a bone. This bone enhancement process is illustrated in Fig. 2.

The modified sheetness score successfully identifies and enhances thin structures. Unfortunately, it also enhances noise and artifacts and its use alone will not produce correct results. In the following section, we present a threshold-based strategy that allows the extraction of the entire bony structure, while eliminating most of the enhanced noise.

\subsection{Bony structure segmentation}

Most voxels of a typical CBCT human skull dataset are either background, air or soft tissue. Therefore, it is desirable that such unnecessary data be filtered out prior to segmentation. We use the sheetness score and a threshold approach to filter all bony structures, reducing the amount of unnecessary data as much as possible. This filter is defined as follows:

$\Omega_{i}^{\prime}=\left\{\begin{array}{ll}-1000, & \text { if } \Omega_{i} \times S_{i} \leq T \\ \Omega_{i} \times S_{i} & \text { otherwise }\end{array}\right.$,

where $\Omega_{i}^{\prime}$ is a new HU value computed for each voxel, $T$ is a threshold parameter and $\Omega_{i}$ and $S_{i}$ are the original $\mathrm{HU}$ and sheetness score, respectively. The value $\Omega_{i}=-1000$ is assigned to voxels that do not belong to a bone, since it is the shortest $\mathrm{HU}$ value in our CBCT data in the range $[-1000,5669]$. This bony structure extraction is illustrated in Fig. 3.

\subsection{Super-voxels}

The aforementioned filter substantially reduces the number of pixels to be processed. However, further reduction can and should be performed to accelerate segmentation. Supervoxel (called super-pixels in 2D image processing) is the outcome of a region-based segmentation technique which groups a set of voxels that share a common image property [9]. They capture redundancy in images and reduce the complexity of subsequent processing tasks [1]. There exist different approaches for super-pixels [2], but not all of them can be easily adapted to the 3D super-voxel scenario. We build on the idea of a 2D super-pixels strategy called speededup turbo pixels (SUTP) [8] that is efficient and simple. It produces high-quality super-pixels, reducing resolution by around $90 \%$ [9].

The 3D version of SUTP, called speeded-up turbo voxels (SUTV), is based on the $k$-means clustering algorithm. First, the image is divided into $N$ regular super-voxels $\Omega_{I}^{\prime}(I \in$ $N$ ), or cubes, with edge length $l$. Initially, all super-voxels, which contain a subset of the original voxels, have the same volume and equidistant centroids. For simplicity, we assume that the minimal bounding box of the entire 3D image dataset is itself a cube. In subsequent processing steps, voxels along a certain super-voxel boundary are analyzed and possibly shifted across the border between neighboring super-voxels, based on the minimization of the following cost function:

$C_{i}=p_{1}\left|\Omega_{i}^{\prime}-\Omega_{I}^{\prime}\right|+p_{2}\left|\left(x_{i}-x_{I}\right)^{2}+\left(y_{i}-y_{I}\right)^{2}+\left(z_{i}-z_{I}\right)^{2}\right|$,

where $\Omega_{i}^{\prime}$ is the HU value of the voxel under consideration, $\Omega_{I}^{\prime}$ is the mean $\mathrm{HU}$ value of the $I$ th super-voxel, $\{x, y, z\}_{i}$ is the location of the tested voxel, and $\{x, y, z\}_{I}$ is the centroid of the $I$ th super-voxel. The parameters $p_{1}$ and $p_{2}$ are weights for HU similarity and super-voxel border rigidity, respectively. Figure 4 shows a CBCT slice and the super-voxels after convergence. Notice how well- adjusted super-voxels are to the bone contours.

Parameters $p_{1}, p_{2}$ and the number of iterations were extensively analyzed in [10] in a 2D scenario, following the original equation proposed in [8]. The authors suggest the values $p_{1}=1$ and $p_{2}=0.5$, with $i t=5$ (it being the number of iterations) for super-pixels with size $l$ in the range $[10-15]$. Given the similarity between super-voxel Eq. 4 and the original one, we set the super-voxel parameters $p_{1}$, $p_{2}$ and $i t$ as suggested in [10]. A thorough analysis of optimal values for parameter $l$ is provided in Sect. 4.1.

\subsection{Seed-based graph cut segmentation}

Three-dimensional segmentation can be considered a voxel labeling problem. In general, the labels of the voxels defining an object of interest are set to 1 (foreground), while all other voxels' labels are set to 0 (background). Final segmentation is generated by minimizing an energy function [36]. 
Fig. 4 Super-voxel convergence after five iterations. a Bone structure. b Super-voxels and the palatine bone in a yellow box frame. c Close-up view of (b). d 3D view

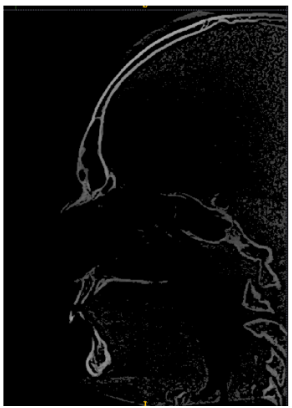

(a)

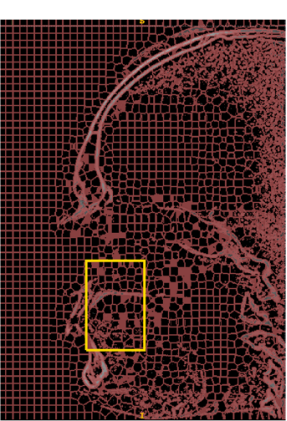

(b)

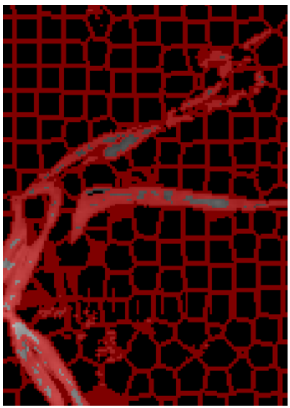

(c)

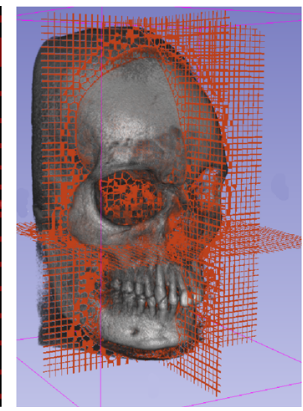

(d)
Usually, a graph is constructed so that each voxel corresponds to a vertex, using edge weights based on a weight function applied to edge-connected vertices [26]. Although graph cut algorithms are efficient and can produce a global optimum for the energy function, they are limited in practice due to the complexity of the graph [36]. A typical graph representing a 3D image dataset consists of several million vertices, with unacceptable graph processing times and memory requirements. By using super-voxels during segmentation, we can reduce the number of vertices up to $90 \%$.

Let $G=\left(V, E, W_{E}\right)$ denote a weighted graph, where $V$ is the set of vertices defining super-voxel $\Omega_{I}, E$ is the set of edges defining pairs of connected super-voxels and $w_{I J} \in W_{E}$ is the weight assigned to an edge $\left(\Omega_{I}, \Omega_{J}\right)$. Ideally, edge weights represent best possible similarity values between two connected vertices. Commonly, weights are computed according to some image feature, e.g., color, intensity, gradient magnitude or contours [26]. Nonetheless, voxels belonging to the same bone can have different intensity values. On the other hand, regions of different bones may share similar textures. Thus, weights considering both features could lead to a graph with low edge weights for voxels of the same bone. We compute edge weights based on a Laplacian function and the spatial location of super-voxels' centroids as follows:

$w_{I J}=\exp \left(-\frac{\sqrt{\left(x_{I}-x_{J}\right)^{2}+\left(y_{I}-y_{J}\right)^{2}+\left(z_{I}-z_{J}\right)^{2}}}{\delta}\right)$,

where $\{x, y, z\}_{I}$ is the centroid of the $I$ th super-voxel, $J \in$ $V_{I}$, with $V_{I}$ being the set of adjacent super-voxels. The Laplacian function leads to low weights for adjacent super-voxels with centroids that are spatially distant from each other. We compute the parameter $\delta$ as follows:

$$
\delta=\max _{J \in V_{I}} \sqrt{\left(x_{I}-x_{J}\right)^{2}+\left(y_{I}-y_{J}\right)^{2}+\left(z_{I}-z_{J}\right)^{2}} .
$$

Notice that intensity is not considered in Eq. 5, as all supervoxels under investigation belong to a bone. Thus, intensity would not contribute to a better data discrimination.

Once the graph has been constructed, we employ a binary labeling strategy by computing the global minimum of an energy function $E(\mathbf{x})$ [5]. Let $B$ and $F$ be the sets of background and foreground voxels, respectively. Let $q_{B}$ and $q_{F}$ be the seed labels of background and foreground super-voxels assigned during the energy optimization process, defined as follows:

$E(\mathbf{x})=\mathbf{x}^{\mathbf{t}}\left(\mathbf{I}+\mathbf{L}^{\mathbf{2}}\right) \mathbf{x}-2 \mathbf{x}^{\mathbf{t}} \mathbf{b}+c$,

where $\mathbf{I}$ is the identity matrix; $\mathbf{L}$ is the Laplacian matrix of the graph; $c$ is a constant; and $\mathbf{b}$ is a vector in which

$\mathbf{b}_{I}=\left\{\begin{array}{ll}q_{B}, & \text { if } I \in B \\ q_{F}, & \text { if } I \in F \\ 0, & \text { otherwise }\end{array}\right.$.

Since $\mathbf{I}+\mathbf{L}^{\mathbf{2}}$ is a symmetric and positive definite matrix, minimization of the energy $E(\mathbf{x})$ can be achieved by solving a linear system of equations, given as

$\left(\mathbf{I}+\mathbf{L}^{\mathbf{2}}\right) \mathbf{x}=\mathbf{b}$

with $\mathbf{x}$ being the solution. This system can be efficiently solved with Cholesky factorization. As a result, the foreground and background labels $r_{I} \in\left\{q_{B}, q_{F}\right\}, I \in V$, are assigned as follows [5]:

$r_{I}=\left\{\begin{array}{ll}q_{B}, & \text { if } q_{i} \geq \frac{q_{B}+q_{F}}{2} \\ q_{F}, & \text { otherwise }\end{array}\right.$.

\section{Method}

The diagram of Fig. 5 illustrates the interactive graph-based segmentation method of CBCT of human skull proposed in this paper. 


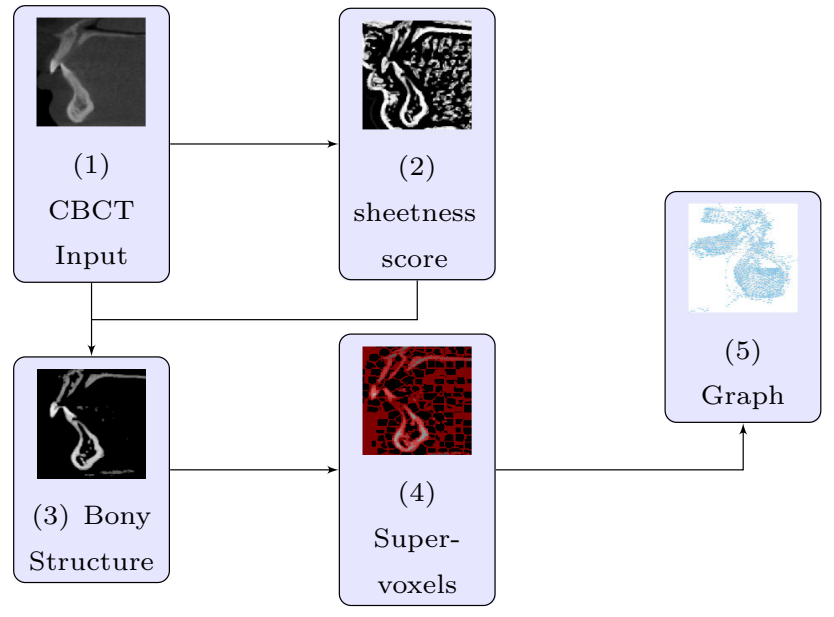

(a)

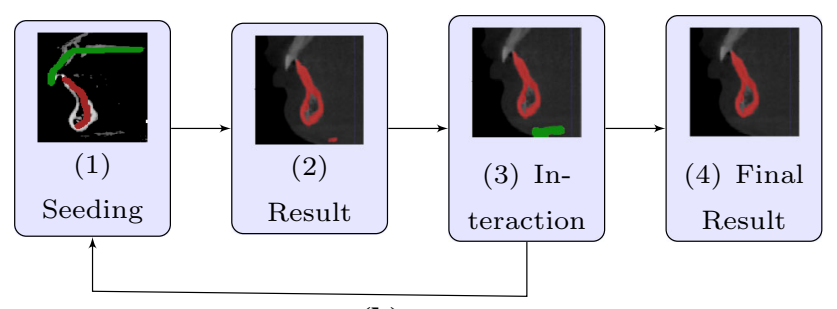

(b)

Fig. 5 Interactive two-stage segmentation of CBCT: a preprocessing. Automatic segmentation of bony structures, followed by grouping of similar voxels into super-voxels and graph representation where each vertex is a super-voxel; b interactive segmentation. User selects the bone of interest by placing seeds on it (brush strokes). Segmentation can be improved by either adding or removing seeds interactively. a Automatic pre-segmentation of bony structures. b Interactive Segmentation: bone of interest marked with a red brush stroke; the green stroke is the undesired region. The red region in (2), (3) and (4) is the resulting segmentation

The first stage of our approach consists in automatically building a graph from the bony structure. We first extract the entire bony structure with the bone enhancement algorithm (sheetness score) introduced in Sect. 2.1. This allows the accurate identification of thin bones such as the maxilla. Following the bone enhancement, we apply a threshold strategy to separate bones from tissue, air and image background (Sect. 2.2). From the bony structure, we finally build a graph whose vertices represent super-voxels (Sects. 2.3, 2.4).

The second stage is an interactive seed-based segmentation process. By applying brush strokes on any of the three image planes (axial, sagittal and coronal), a user can select bones of interest (red strokes for foreground) and undesired structures (green strokes for background).

The super-voxels comprised in the brush strokes form the input vectors $\mathbf{b}$ of the linear system given in Equation 9. The graph is then segmented into two sets (foreground and background) using the Cholesky decomposition (Eq. 10).
Segmentation can be further improved by continuous interaction with the addition or removal of seeds. This seeding strategy allows seeds to be placed on thin internal bones, which is important for the identification of small details. Bare in mind that the first stage of our approach is executed only once, whereas the second interactive process can be applied until the desired segmentation is attained.

Selecting internal thin bones with brush strokes on 2D planes can be a delicate task. This may cause undesired regions (adjacent tissue) to be seeded accidentally. However, the pre-segmentation process allows the accurate extraction of all bony structures, including thin bones. As a result, seeds can be placed on either adjacent tissue, air or image background without compromising the segmentation quality. That is, there is no need for an accurate placement of strokes as long as undesired bones are not seeded. This renders a fast, robust and accurate interactive segmentation process. Nevertheless, to guide the selection of a bone of interest, we recommend seeding at least one slice per plane (sagittal, coronal and axial plane). Seeds should be placed over areas such as low-contrast regions of the maxilla, or bones close to each other, the condyle, for example.

Graph-based segmentation strategies directly applied on unprocessed $\mathrm{CBCT}$ data will certainly have to deal with a huge number of vertex. Graph cut algorithm, for example, would not only take far too long to process, but also demand large memory resources. Reducing processing time and memory requirements is mandatory for any feasible interactive segmentation approach. Therefore, we reduce the graph cardinality by minimizing the quantity of redundant similar voxels, grouping them into super-voxels. This clustering task contributes to reduce redundant information up to $90 \%$, making the interactive segmentation friendly and computationally feasible.

\section{Results and validation}

We employed an empirical discrepancy approach to validate the results generated by our method. We compared our results with the best expected segmentation produced (ground truth, GT) [38]. Our GTs were manually created by experienced orthodontists using the ITK-Snap tool whose output is a mesh model. To compare with our voxel-based segmentation method, we had to compute a similar representation. We chose the marching cubes algorithm [19] to generate a similar mesh model, the same as used in ITK-Snap. We measured the similarity between two meshes using the Jaccard index and Dice coefficient [18]. Our CBCT data have a $400 \times 400 \times 554$ resolution with an isotropic voxel size of $0.4 \mathrm{~mm}$ of 17-year-old patients (male and female), acquired with an i-Cat scanner. 
Although automatic, the pre-segmentation stage of our proposed framework requires three parameters: (i) a Gaussian standard deviation $\sigma$ used for the sheetness score; (ii) a threshold $t$, for bony structure extraction; and (iii) an initial side length $l$ of each super-voxel. In general, setting up these parameters is a time-consuming task imposed on users. In Sect. 4.1, we present an analysis where parameter settings were evaluated in order to define an appropriate setup.

We also compared our seed-based interactive segmentation method with a related segmentation approach described by [40]. Results are provided in Sect. 4.2. Finally, we tested our approach for ordinary CT data, publicly available. Results are presented in Sect. 4.3.

\subsection{Parameter optimization}

We segmented the mandible and skull of sixteen datasets, for which GT data were also available (eight mandible GTs and eight skull GTs). Again, the pre-segmentation stage demands three parameters: $\sigma, t$ and $l$. We evaluated the parameters in the following ranges: $\sigma$ from 0.5 to 1.0 (step size 0.1 ); threshold $t$ from 100 to 300 (step size 50); and super-voxel edge length $l$ from 10 to 15 (step size 1) and 5 iterations. As a result, 180 segmented meshes were produced for each bone. We compared all results with their corresponding GTs. Regarding the parameter value ranges mentioned above, best segmentation accuracy, for both skull and mandible, can be obtained with the parameter values shown in Table 1.

Figure 6 shows an example of the seed placement process and the resulting 3D segmentation for two datasets. In the following subsections, we provide more details about the segmentation of the skull and the mandible.

\subsubsection{Skull segmentation}

The plots shown in Fig. 7 represent segmentation accuracy (Jaccard curves) for different values of the three parameters. Best segmentation was observed for threshold $(t)$ values within the range $150 \leq t \leq 300$. However, in the presence of thin bones, which are the most challenging structures to segment, the sheetness score $(\sigma)$ plays a crucial role. Figures 8 and 9 show how different values of $\sigma$ and $t$ affect the segmentation of sheetlike bones. Regarding initial super-voxel side length $l$, best results were obtained with $10 \leq l \leq 12$. For this range of $l$ values, super-voxels are well adjusted to borders after only 5 iterations. Larger super-voxel sizes demand more iterations for convergence. The mean and standard deviation values for all datasets are listed in Table 2.

\subsubsection{Mandible segmentation}

Unlike the skull, the mandible does not comprise several thin bones, albeit it has some structures, such as coronoid process
Table 1 Parameter values for most accurate segmentation

\begin{tabular}{l}
\hline Parameter intervals \\
\hline $0.7 \leq \sigma \leq 1.0$ \\
$150 \leq t \leq 300$ \\
$10 \leq l \leq 12$ \\
\hline
\end{tabular}

and condyle, with prevailing low contrast causing diffuse borders in images. Segmentation of such bone parts is difficult. Nonetheless, the sheetness score enhances sheetlike structures as well as low-contrast borders, leading to correct segmentation of such bone regions. Plots (Jaccard curves) of segmentation accuracy for different values of $t, \sigma$ and $l$ are shown in Fig. 10. Figures 11 and 12 illustrate the segmentation of coronoid process and condyle for different parameter configurations. The mean and standard deviation values for all datasets are listed in Table 3.

We quantitatively analyzed the influence of the parameters used in the first stage of our method. Results show that optimal results (Jaccard $\approx 0.9$ ) can be obtained with the settings listed in Table 1. Besides, we observed that the most influential parameters are $\sigma$ and $t$, for which a correct setup ensures a good extraction of the entire bony structure, whereas $l$ has less impact on the segmentation quality, because supervoxels converge quickly to object boundaries (5 iterations for $10 \leq l \leq 15$ ). Using these settings, in our experiments, the best results were obtained with $\sigma=0.8, t=200$, and $l=10$.

\subsection{Comparison with related work}

We compared our approach with two related segmentation methods: Zhu et al. [40] and Pauchard et al. [24]. The former is based on a reformulated GrowCut segmentation method with an adaptive version of the Dijkstra algorithm which employs user-placed seeds. The latter is a version of the Graph cut-based segmentation method by Boykov et al. [3]. This method uses only user-placed seeds as object background information, instead of traditional statistical information such as histograms. ${ }^{1}$

Both methods are also interactive and use manually placed seeds for region-of-interest selection (background and foreground). However, they are computationally expensive and require a high amount of memory. Consequently, segmentation must be carried out locally. Zhu et al.'s method automatically defines an axis-aligned bounding box, encompassing all seeds, whereas Pauchard et al.'s method asks users to manually define a region of interest (usually a bounding box).

\footnotetext{
${ }^{1}$ We used the implementation provided by the authors, which includes its own user interface.
} 


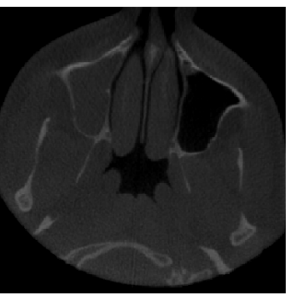

(a)

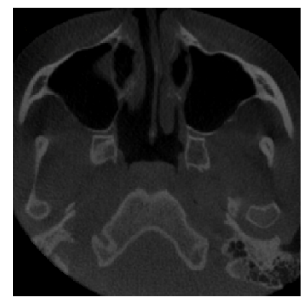

(e)

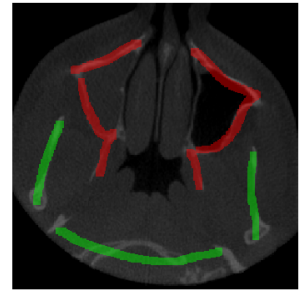

(b)

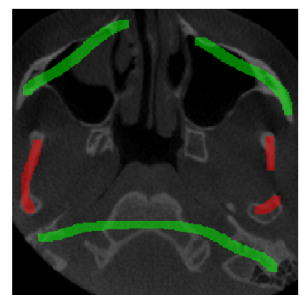

(f)

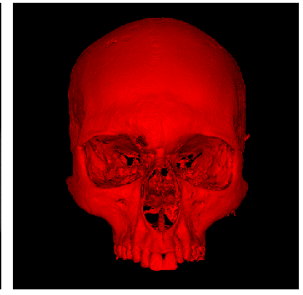

(c)

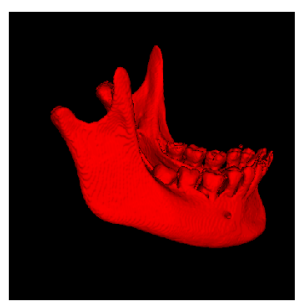

(g)

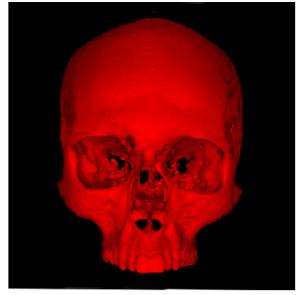

(d)

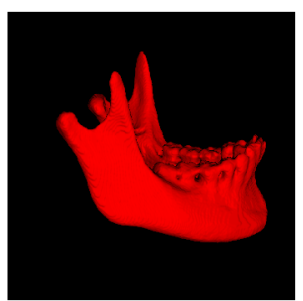

(h)

Fig. 6 Upper row: segmentation of skull of male patient (including mandible). Lower row: segmentation of mandible of female patient. From left to right: axial samples, seed placement process, 3D view of final segmentation, and 3D view of respective ground truth. Red brush strokes indicate the bone of interest (foreground), and green strokes label undesired bones (background). Brush strokes do not have to be

precise and may be drawn beyond bone boundaries, as long as they do not touch other bones. a Dataset 1 (male). b Seeds for skull segmentation. c Resulting skull segmentation. d Ground truth. e Dataset 2 (female). f Seed placement for mandible segmentation. $\mathbf{g}$ Resulting mandible segmentation. $\mathbf{h}$ Ground truth

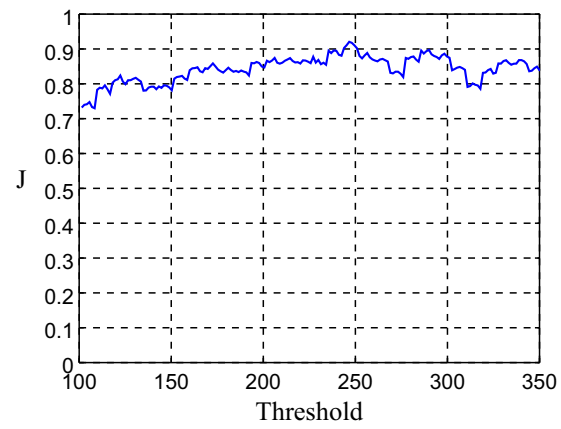

(a)

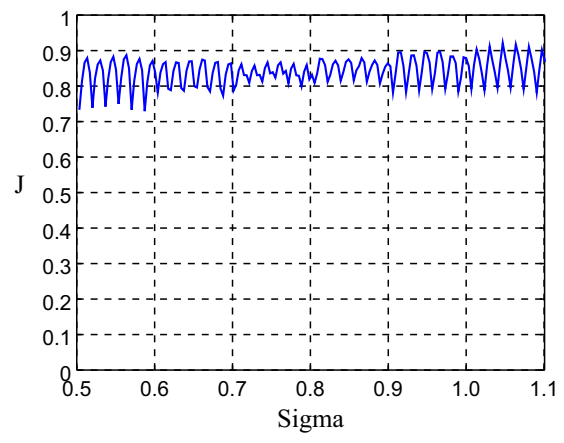

(b)

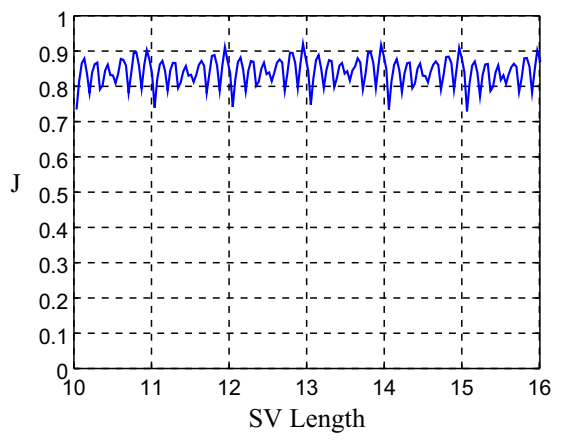

(c)
Fig. 7 Influence of parameters $t, \sigma$ and $l$ on skull segmentation quality. The curves represent the mean Jaccard index of the eight datasets (1440 segmentation results). We measured the segmentation quality by evaluating several combinations of the parameters mentioned, for instance: $t=100, \sigma=0.5$ and $t=[100-300]$. The most influential parameter is the threshold (a) where better qualities are obtained for $t>150$. However, $\sigma$ and $l$ also affect, minimally, the segmentation quality, visible as minor fluctuations in the curves (b) and (c), respectively
We used similar seeding setup conditions and same GT data to segment the mandible of four datasets. Figure 13 shows an example of the seeds we have used to segment the mandible with our method and the result of Zhu's method using the same seeds. Still in Fig. 13, we show results for Pauchard's method, which required more precise seeds to produce an acceptable mandible segmentation. In all cases, our method produced better results than Zhu and Pauchard. In general, segmentation can be improved by placing more precise seeds, thus demanding extra user interaction and steps. However, there are some regions for which Zhu' and Pauchard's methods do not produce accurate segmentation. Zhu presents several "leaks" to adjacent tissue, especially in low-contrast regions, whereas Pauchard does not fit accurately to the mandible contours producing a rough segmentation. Table 4 shows the respective Jaccard index and Dice coefficient.

We also evaluated the methods for a thin bone scenario, with the segmentation of the skull. In general, segmentation of thin structures poses a challenge for seed-based approaches. It is difficult to constrain the strokes to foreground regions only. Voxels belonging to adjacent tissues or background areas can be included by mistake. Thin bones have low-intensity values, and wrong segmentation is likely to occur, even though when seeds are correctly placed. These are the main reasons why Zhu and Pauchard were not able 


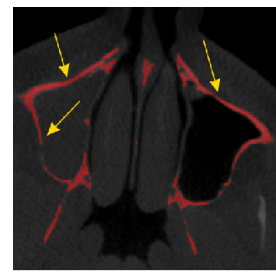

(a)

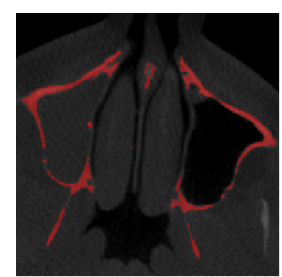

(d)

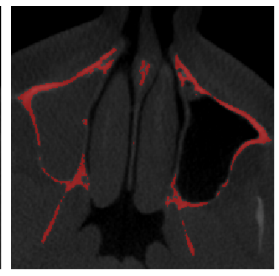

(b)

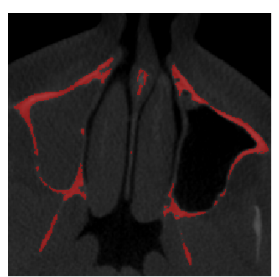

(e)

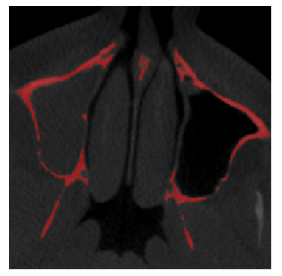

(c)

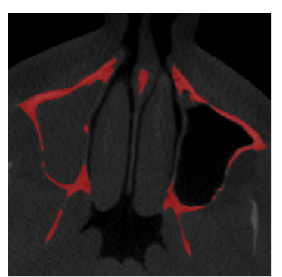

(f)
Fig. 8 Axial view of skull segmentation of the maxilla for different values of $\sigma$, with $t=200$ and $l=10$. Sheetlike bones, such as the anterior and posterior walls of the sinus [yellow arrows in (a)], are accurately segmented regardless of changes in $\sigma$ value. $J$ is the Jaccard index. a $\sigma=0.5, J=0.793$. b $\sigma=0.6, J=0.852$. c $\sigma=0.7$, $J=0.900$. d $\sigma=0.8, J=0.950$. e $\sigma=0.9, J=0.917$. f Ground truth

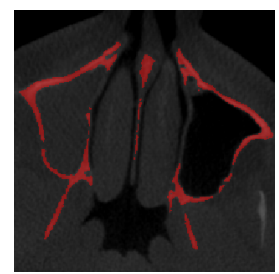

(a)

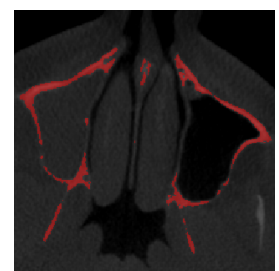

(d)

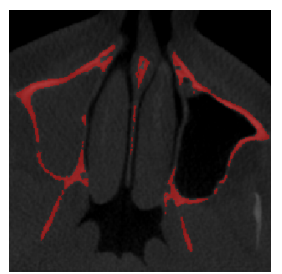

(b)

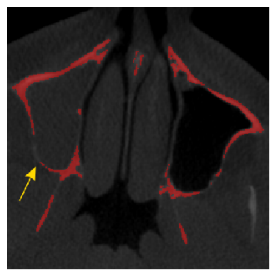

(e)

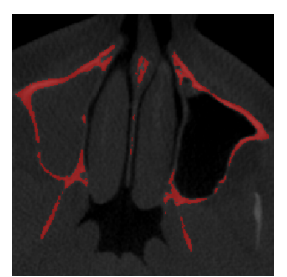

(c)

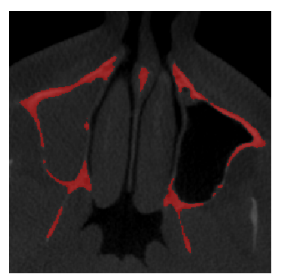

(f)
Fig. 9 Axial view for skull segmentation of the maxilla for different values of $t$, with $\sigma=0.8$ and $l=10$. Changes in threshold $t$ affects segmentation quality, unlike variations in $\sigma$ (see Fig. 8). The retroantral fat pad [yellow arrow in (e)] is not entirely segmented for $t \geq 250$. a $t=100, J=0.809$. b $t=150, J=0.866$. c $t=200, J=0.930$. d $t=250, J=0.950$. e $t=300, J=0.930$. f Ground truth to correctly segment the skull. Our approach, however, is not impaired in the same way. The pre-segmentation stage guarantees robust algorithmic behavior even though when seeds are not perfectly placed and bones have low-intensity values. A skull segmentation example is shown in Fig. 14.

\subsection{Experiment with public CT data}

We also used a publicly available CT data to evaluate our method [27]. No GT data were available to perform a quantitative analysis. Nevertheless, experienced specialists empirically confirmed the high quality of our results. Figure 15 shows a skull segmentation of a patient after surgical repair of facial deformity. The extraction of the mandible of a patient with missing teeth is shown in Fig. 16.

Manually segmentation of broken or fractured bones usually is a more complicated task than segmenting regular bones. Therefore, this task may demand expert knowledge and more user interaction time [25]. Nevertheless, our results demonstrate the potential of our method to segment CT and CBCT for a wide range of patients, including patients with facial deformities, broken and missing bones.

Apart from the segmentation results discussed in Sect. 4.1, all other results were obtained with the following parameters: $l=10, \sigma=0.8, t=200$. We performed our experiments on a Linux workstation (Intel Core i7-2600 CPU $3.40 \mathrm{GHz}$ $\times 4$ with 16 GB Memory). The $\mathrm{C}++$ source code included the National Library of Medicine Insight Segmentation and Registration Toolkit (ITK) [14] and the C++ template library of the linear algebra library Eigen [13]. All results were rendered with ITK-Snap.

\section{Conclusions}

We have introduced a new interactive method for CBCT data segmentation. Our approach consists of two stages: (i) automatic bone structure extraction and (ii) interactive seed-based bone segmentation. We validated our approach both quantitatively and qualitatively with three experiments: (i) detailed evaluation of the parameter space; (ii) comparison with a related method; and (iii) segmentation of CT data with missing and broken bones.
Table 2 Mean $(\mu)$ and standard deviations $(\sigma)$ of Jaccard index and Dice coefficient for skull segmentation of all datasets

\begin{tabular}{|c|c|c|c|c|c|c|c|c|}
\hline \multirow[t]{2}{*}{ Metric } & \multicolumn{8}{|c|}{$\underline{\text { Skull datasets }}$} \\
\hline & 1 & 2 & 3 & 4 & 5 & 6 & 7 & 8 \\
\hline Jaccard $\mu$ & 0.828 & 0.874 & 0.865 & 0.827 & 0.878 & 0.857 & 0.847 & 0.848 \\
\hline Jaccard $\sigma$ & 0.057 & 0.049 & 0.047 & 0.051 & 0.054 & 0.045 & 0.037 & 0.039 \\
\hline Dice $\mu$ & 0.905 & 0.932 & 0.921 & 0.904 & 0.903 & 0.922 & 0.915 & 0.917 \\
\hline Dice $\sigma$ & 0.034 & 0.028 & 0.025 & 0.030 & 0.033 & 0.026 & 0.034 & 0.031 \\
\hline
\end{tabular}




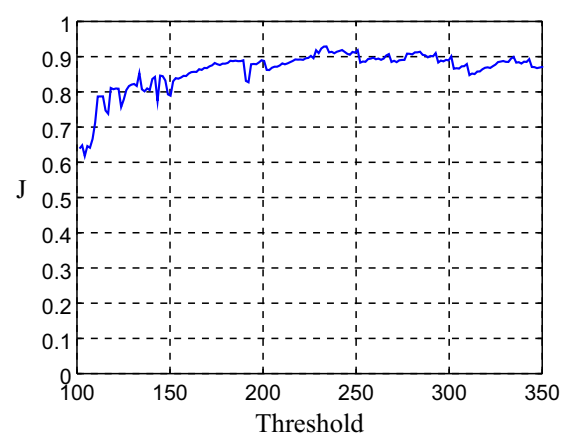

(a)

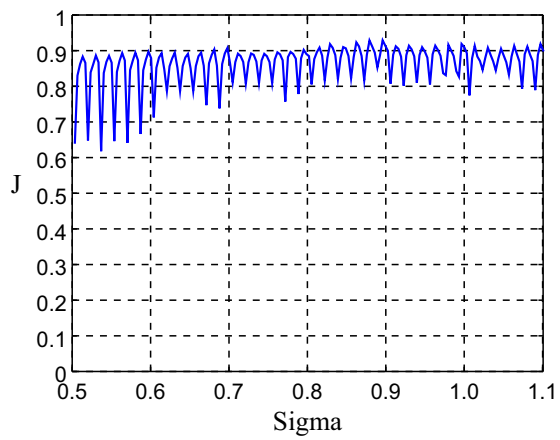

(b)

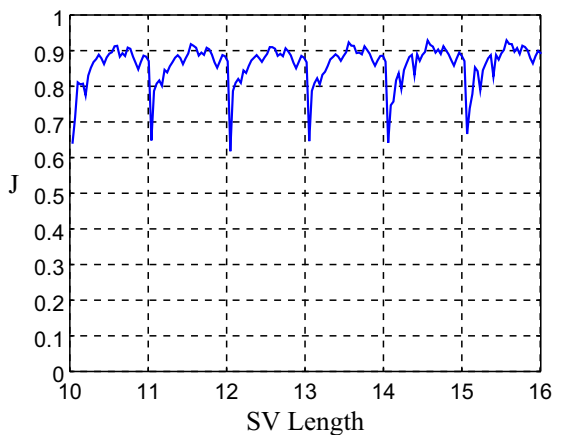

(c)
Fig. 10 Influence of parameters $t, \sigma$ and $l$ on mandible segmentation quality. We produced 180 segmentation of the mandible for eight CBCTs (with a total of 1440 segmentations). Curves show the mean Jaccard index for the eight datasets evaluated. We measured segmentation quality by evaluating several combinations of the parameters, for instance $t=100, \sigma=0.5$ and $t=[100-300]$. The most influential parameter is the threshold [see (a)], where better results are obtained for $150<t<300$. However, $\sigma$ and $l$ also affect, minimally, segmentation quality, visible as fluctuations in the curves. There exist local extrema, e.g., local extrema for $t<150$ [see (a)]; these are explained by the presence of noise in segmentation (see Fig. 12c). It is possible to interactively eliminate it through brush strokes (see Fig. 5), improving segmentation quality. Local extrema are the same in all curves

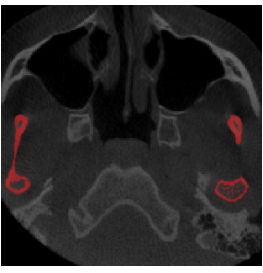

(a)

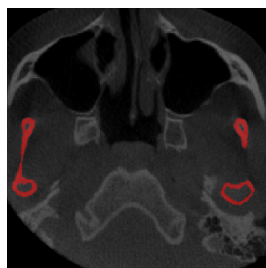

(d)

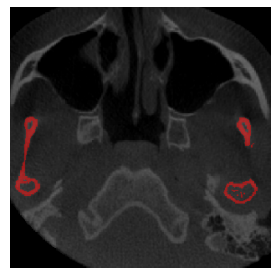

(b)

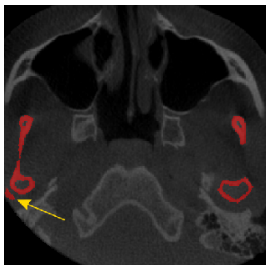

(e)

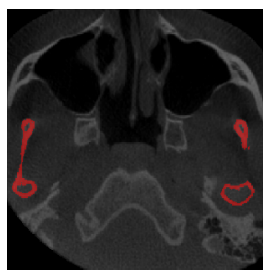

(c)

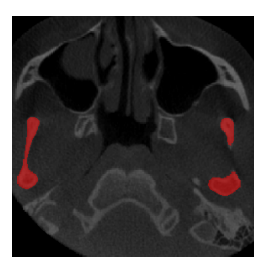

(f)
Fig. 11 Axial view of the mandible segmentation (condyle and coronoid process), using $t=200$ and $l=10$. Segmentations obtained with parameter $\sigma$ at different scales. Better segmentations result for $\sigma \geq 0.7$. Noise can appear in segmentations [yellow arrows in (e)], which could interactively be eliminated with additional brush strokes (see Fig. 5). a $\sigma=0.5, J=0.817$. b $\sigma=0.6, J=0.873$. c $\sigma=0.7, J=0.924$. d $\sigma=0.8, J=0.950$. e $\sigma=0.9, J=0.909$. f Ground truth

Our results document that our method produces accurate segmentations of both skull and mandible. Contours affected by low contrast are precisely segmented (condyle or coronoid process), and thin bones (maxilla) were also properly segmented. Our method exhibits low sensitivity to parameter value settings and does not require precise seed placement. Moreover, our method robustly handles low contrast, noisy and inhomogeneous data. Segmentation is done in approximately 5 min (both stages), on average, which is considerably faster compared to the efficiency of similar segmentation

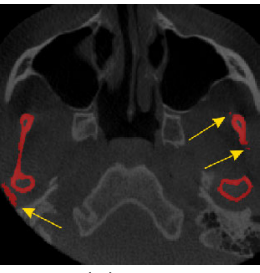

(a)

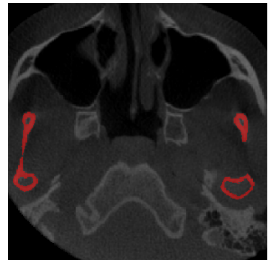

(d)

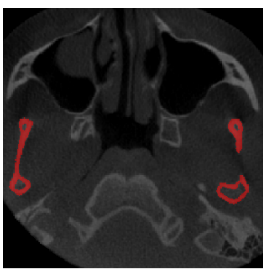

(b)

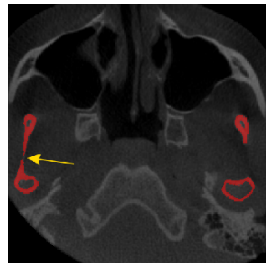

(e)

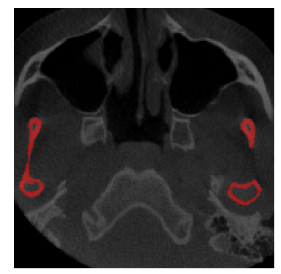

(c)

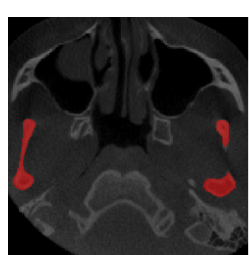

(f)
Fig. 12 Axial view of mandible segmentation (condyle and coronoid process ), using $\sigma=0.8$ and $l=10$. Segmentations shown for different values of threshold $t$. Higher quality is achieved for $100<t<300$. Higher values of $t$ do not yield proper segmentation of thin bone regions, e.g., the coronoid process [yellow arrow in (e)]. Noise can result in the final segmentation [yellow arrows in (a)] for $t<100$. One can interactively eliminate it through brush strokes. a $t=100, J=0.909$. b $t=150, J=0.956$. c $t=200, J=0.950$. d $t=250, J=0.963$. e $t=300, J=0.937$. f Ground Truth

methods. Our method is clearly much faster than manual segmentation.

We emphasize that the first stage, which accounts for $80 \%$ of total computing time, is performed only once. The second stage, although interactive, takes on average about 1-2 min. It can also be repeated many times to fine-tune segmentation.

The processing time of $5 \mathrm{~min}$ is the average time needed to segment either the mandible or the skull in a CBCT sample 
Table 3 Mean $(\mu)$ and standard deviations $(\sigma)$ of Jaccard index and Dice coefficient of mandible segmentation

\begin{tabular}{lllllllll}
\hline Metric & \multicolumn{7}{l}{ Mandible datasets } & \multicolumn{7}{l}{ ( } & 2 & 3 & 4 & 5 & 6 & 7 & 8 \\
\cline { 2 - 8 } & 1 & 0.851 & 0.882 & 0.854 & 0.858 & 0.878 & 0.869 & 0.873 \\
Jaccard $\mu$ & 0.853 & 0.093 & 0.051 & 0.104 & 0.053 & 0.042 & 0.068 & 0.046 \\
Jaccard $\sigma$ & 0.108 & 0.929 & 0.936 & 0.917 & 0.912 & 0.934 & 0.939 & 0.947 \\
Dice $\mu$ & 0.916 & 0.929 & 0.029 & 0.073 & 0.012 & 0.023 & 0.025 & 0.036 \\
Dice $\sigma$ & 0.076 & 0.059 & 0.029 &
\end{tabular}

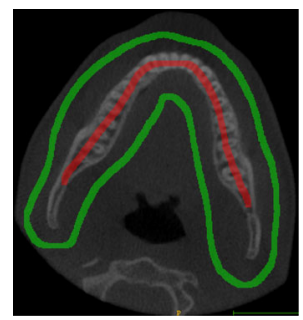

(a)

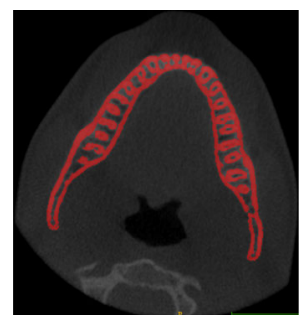

(b)

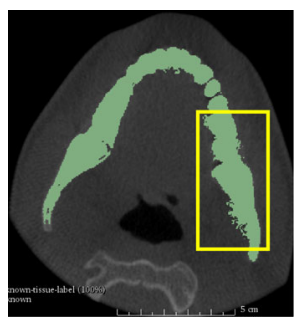

(c)

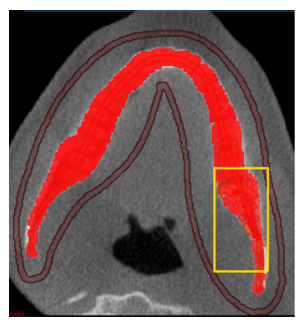

(d)
Fig. 13 Axial view of mandible segmentation obtained by our method, Zhu et al.'s method, and Pauchard et al.'s method. Similar seeding setup (a), with red indicating the desired bone and green undesired regions; $\mathbf{b}-$ d show corresponding segmentation results. Zhu et al.'s results (green) exhibit "leaks" (yellow frame) in low-density areas. Several more seeds

Table 4 Comparing our method with Zhu et al.'s and Pauchard et al.'s in a mandible segmentation scenario

\begin{tabular}{lllll}
\hline & Mandible 1 & Mandible 2 & Mandible 3 & Mandible 4 \\
\hline Our method & 0.92 & 0.93 & 0.94 & 0.93 \\
Zhu & 0.88 & 0.87 & 0.88 & 0.89 \\
Pauchard & 0.89 & 0.90 & 0.91 & 0.90 \\
\hline
\end{tabular}

Values are the Jaccard similarity with respect to ground truth

of our dataset. This processing time includes the seeding step (one iteration) and segmentation processing. Our implementation of the super-voxel algorithm uses a data structure we designed that allows one to access super-voxel boundaries in constant time, i.e., there is no need for searching. Generating

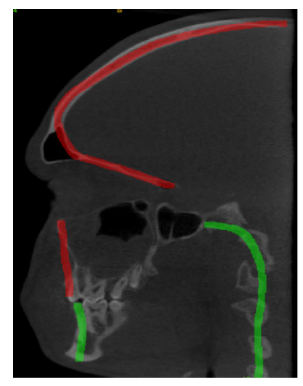

(a)

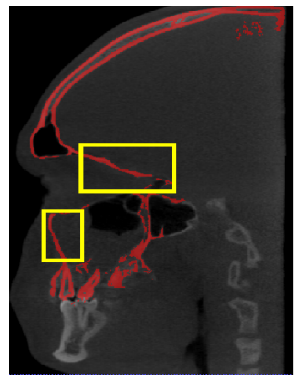

(b)

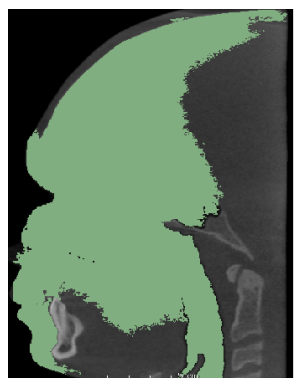

(c)
Fig. 14 Sagittal view of skull segmentation obtained by our approach and by Zhu et al.'s method. The seeding setup is shown in (a), with red indicating the desired bone and green undesired regions. Our method properly segments thin bones, even though when brush strokes cover

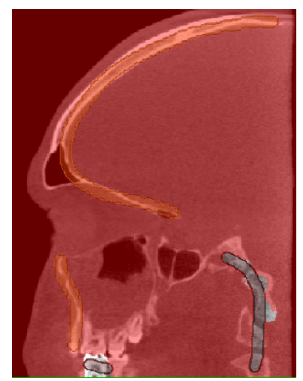

(d) are required to produce an "acceptable" segmentation (red region) when using Pauchard et al.'s method. Despite the use of more seeds, the segmentation does not properly fit mandible borders. a Seed placement. b Ours. c Zhu et al. d Pauchard et al.

super-voxels for a typical CBCT sample takes about 2-3 min. Memory usage is about $10 \mathrm{GiB}$. The short user interaction time is due to the robustness of our method to imprecise seed placement.

The robustness of our method with respect to imprecise seed placement is a consequence of the pre-segmentation step, which enhances and pre-segments bones, especially thin ones. Once bones are separated from other tissue types and structures, strokes drawn outside bone regions, i.e., strokes wrongly placed in non-bone regions, have no negative effect on segmentation quality. The use of super-voxels to group voxels of similar bone structures drastically reduces graph cardinality (up to $90 \%$ ) and makes it possible to employ a precise, but computationally expensive, linear system

adjacent tissue types [see yellow frames in (b)]. Zhu et al.'s method produces "leaks" in soft tissue regions and air regions (cranial cavities). Pauchard et al.'s method leaks into the image background (red region). a Seed placement. b Ours. c Zhu et al. Pauchard et al. 


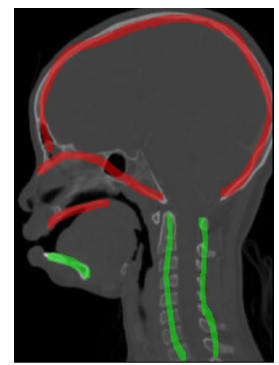

(a)

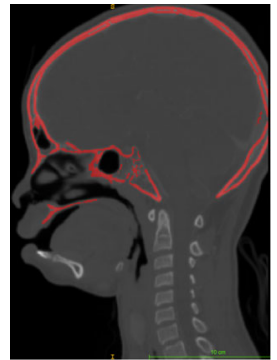

(b)

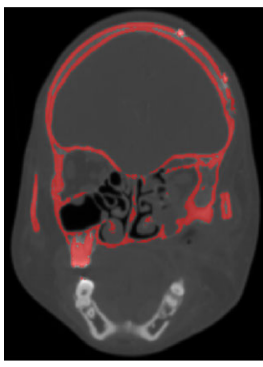

(c)
Fig. 15 Skull segmentation. CT of patient with facial deformities after surgical repair. CT dataset of $512 \times 512 \times 361$ resolution and anisotropic voxel spacing of $0.42,0.42$ and $0.70 \mathrm{~mm}$ in the three directions. a Seed placement. b Sagittal view. c Coronal View

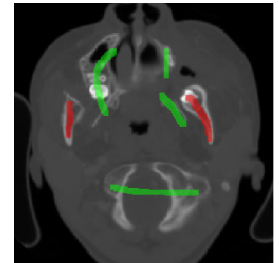

(a)

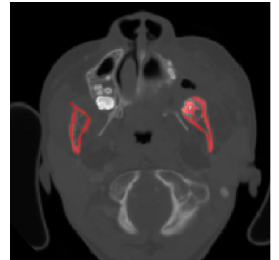

(b)

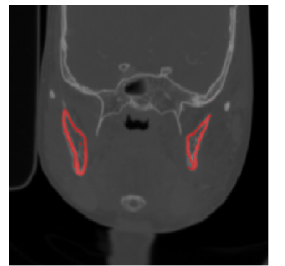

(c)
Fig. 16 Segmentation of mandible. Patient with missing teeth. CT dataset of resolution $512 \times 512 \times 460$ and anisotropic voxel spacing of $0.48,0.48$ and $0.70 \mathrm{~mm}$ in the three directions. a Seed placement. b Coronal view of masseter. c Axial view of masseter and coronoid process

solver using Cholesky factorization. This strategy reduces the amount of memory required for computation, and it also speeds up the segmentation process, making the interactive process feasible in a clinical environment. Our results show that our approach accurately segments the mandible and skull of various patient types, i.e., male, female, child, and of patients with broken, anomalous or missing bones.

Separating upper and lower teeth, in a closed-mouth situation, is and remains a challenging problem. Our method is already capable of separating them by using a few additional brush strokes (see Fig. 6c). However, visual inspection reveals that accuracy is lower for teeth regions than one would like. We intend to improve the performance of our method to produce high-quality segmentations of teeth as part of our future research.

Acknowledgements This work was supported by the Brazilian research agency Sao Paulo Research Foundation (FAPESP) Grant Numbers: 2012/24036-1 and 2015/12771-7.

\section{References}

1. Achanta, R., Shaji, A., Smith, K., Lucchi, A., Fua, P., Süsstrunk, S.: Slic superpixels. Technical report, cole polytechnique fdrale de Lausanne (2010)
2. Achanta, R., Shaji, A., Smith, K., Lucchi, A., Fua, P., Süsstrunk, S.: Slic superpixels compared to state-of-the-art superpixel methods. IEEE Trans. Pattern Anal. Mach. Intell. 34, 2274-2282 (2012)

3. Boykov, Y., Funka-Lea, G.: Graph cuts and efficient ND image segmentation. Int. J. Comput. Vis. 70(2), 109-131 (2006)

4. Brandariz, M., Barreira, N., Penedo, M.G., Suárez-Cunqueiro, M.: Automatic segmentation of the mandible in cone-beam computer tomography images. In: 27th IEEE International Symposium on Computer-Based Medical Systems, pp. 467-468 (2014)

5. Casaca, W., Nonato, L.G., Taubin, G.: Laplacian coordinates for seeded image segmentation. In: IEEE Conference on Computer Vision and Pattern Recognition, pp. 384-391 (2014)

6. Chang, Y.B., Xia, J.J., Yuan, P., Kuo, T.H., Xiong, Z., Gateno, J., Zhou, X.: 3d segmentation of maxilla in cone-beam computed tomography imaging using base invariant wavelet active shape model on customized two-manifold topology. J. X-ray Sci. Technol. 21, 251-282 (2013)

7. Chung, F., Schmid, J., Magnenat-Thalmann, N., Delingette, H.: Comparison of statistical models performance in case of segmentation using a small amount of training datasets. Vis. Comput. 27(2), 141-151 (2011). https://doi.org/10.1007/s00371-010-0536-9

8. Çı̆̆la, C., Alatan, A.A.: Efficient graph-based image segmentation via speeded-up turbo pixels. In: IEEE International Conference on Image Processing, pp. 3013-3016 (2010)

9. Cuadros Linares, O., Botelho, G., Rodrigues, F., Neto, J.B.: Segmentation of large images with complex networks. In: IEEE 25th SIBGRAPI Conference on Graphics, Patterns and Images, pp. 2431 (2012)

10. Cuadros Linares, O., Botelho, G.M., Rodrigues, F.A., Neto, J.B.: Segmentation of large images based on super-pixels and community detection in graphs. IET Image Process. 11, 60-80 (2017)

11. Descoteaux, M., Audette, M., Chinzei, K., Siddiqi, K.: Bone enhancement filtering: application to sinus bone segmentation and simulation of pituitary surgery. Comput. Aided Surg. 11, 247-255 (2006)

12. Fedorov, A., Beichel, R., Kalpathy-Cramer, J., Finet, J., FillionRobin, J.C., Pujol, S., Bauer, C., Jennings, D., Fennessy, F., Sonka, M., et al.: 3D slicer as an image computing platform for the quantitative imaging network. Magn. Reson. Imaging. 30, 1323-1341 (2012)

13. Guennebaud, G., Jacob, B., Avery, P., Bachrach, A., Barthelemy, S.: Eigen v3. http://eigen.tuxfamily.org. (2010). Accessed 08 Aug 2017

14. Johnson, H.J., McCormick, M., Ibáñez, L.: The ITK Software Guide, 3rd edn. Kitware Inc., Arlington (2013). http://www.itk. org/ItkSoftwareGuide.pdf. Accessed 16 Aug 2017

15. Kirbas, C., Quek, F.: A review of vessel extraction techniques and algorithms. ACM Comput. Surv. (CSUR) 36, 81-121 (2004)

16. Kolmogorov, V., Zabin, R.: What energy functions can be minimized via graph cuts? IEEE Trans. Pattern Anal. Mach. Intell. 26, 147-159 (2004)

17. Krčah, M., Székely, G., Blanc, R.: Fully automatic and fast segmentation of the femur bone from 3D-CT images with no shape prior. In: IEEE International Symposium on Biomedical Imaging: From Nano to Macro, pp. 2087-2090 (2011)

18. Li, W., Magnotta, V.: New Functionalities for Spherical Demons Registration, pp. 08-10 . Kitware Source - Software Developers Quarterly (2011). https://blog.kitware.com/wp-content/uploads/ 2016/01/kitware_quarterly0111.pdf. Accessed 08 Aug 2017

19. Lorensen, W.E., Cline, H.E.: Marching cubes: a high resolution 3D surface construction algorithm. ACM Siggraph Comput. Graph. 21, 163-169 (1987)

20. Loubele, M., Maes, F., Schutyser, F., Marchal, G., Jacobs, R., Suetens, P.: Assessment of bone segmentation quality of cone-beam CT versus multislice spiral CT: a pilot study. Oral Surg. Oral Med. Oral Pathol. Oral Radiol. Endodontol. 102, 225-234 (2006) 
21. Macia, I.: Generalized computation of gaussian derivatives using ITK. Insight J. 1-14 (2007)

22. Miles, D.A., Danforth, R.A.: A clinician's guide to understanding cone beam volumetric imaging (CBVI). Acad. Dent. Ther. Stomatol. 1-13 (2007). https://www.dentalacademyofce.com/courses/ 1413/PDF/_Clin_Gde_ConeBeam.pdf

23. Mozzo, P., Procacci, C., Tacconi, A., Martini, P.T., Andreis, I.B.: A new volumetric CT machine for dental imaging based on the conebeam technique: preliminary results. Eur. Radiol. 8, 1558-1564 (1998)

24. Pauchard, Y., Fitze, T., Browarnik, D., Eskandari, A., Pauchard, I., Enns-Bray, W., Pálsson, H., Sigurdsson, S., Ferguson, S.J., Harris, T.B., et al.: Interactive graph-cut segmentation for fast creation of finite element models from clinical CT data for hip fracture prediction. Comput. Methods Biomech. Biomed. Eng. 19(16), 1693-1703 (2016)

25. Paulano, F., Jiménez, J.J., Pulido, R.: 3D segmentation and labeling of fractured bone from CT images. Vis. Comput. 30(6), 939-948 (2014). https://doi.org/10.1007/s00371-014-0963-0

26. Peng, B., Zhang, L., Zhang, D.: A survey of graph theoretical approaches to image segmentation. Pattern Recognit. 46, 10201038 (2013)

27. [dataset] Pixmeo-SARL: Osirix dicom image sample sets. http:// www.osirix-viewer.com/resources/dicom-image-library (2016). Accessed Apr 2018

28. Richmond, C.: Sir Godfrey Hounsfield. Br. Med. J. 329, 687 (2004)

29. Scarfe, W.C., Farman, A.G., Sukovic, P.: Clinical applications of cone-beam computed tomography in dental practice. J. Can. Dent. Assoc. 72, 75-80 (2006)

30. Solutions, D.I.M.: Dolphin imaging. http://www.dolphinimaging. com (2017). Accessed Apr 2018

31. Spampinato, C., Pino, C., Giordano, D., Leonardi, R.: Automatic 3D segmentation of mandible for assessment of facial asymmetry. In: IEEE International Symposium on Medical Measurements and Applications Proceedings (MeMeA), pp. 1-4 (2012)

32. von Landesberger, T., Andrienko, G., Andrienko, N., Bremm, S., Kirschner, M., Wesarg, S., Kuijper, A.: Opening up the "black box" of medical image segmentation with statistical shape models. Vis. Comput. 29(9), 893-905 (2013). https://doi.org/10.1007/s00371013-0852-y

33. Wang, L., Chen, K.C., Gao, Y., Shi, F., Liao, S., Li, G., Shen, S.G., Yan, J., Lee, P.K., Chow, B., et al.: Automated bone segmentation from dental CBCT images using patch-based sparse representation and convex optimization. Med. Phys. 41, 043503 (2014)

34. Wang, L., Gao, Y., Shi, F., Li, G., Chen, K.C., Tang, Z., Xia, J.J., Shen, D.: Automated segmentation of dental CBCT image with prior-guided sequential random forests. Med. Phys. 43, 336-346 (2016)

35. Xia, J.J., Gateno, J., Teichgraeber, J.F.: A new clinical protocol to evaluate cranio-maxillofacial deformity and to plan surgical correction. J. Oral Maxillofac. Surg. 67, 2093-2106 (2009)

36. Yi, F., Moon, I.: Image segmentation: a survey of graph-cut methods. In: IEEE International Conference on Systems and Informatics (ICSAI), pp. 1936-1941 (2012)

37. Yushkevich, P.A., Piven, J., Cody Hazlett, H., Gimpel Smith, R., Ho, S., Gee, J.C., Gerig, G.: User-guided 3D active contour segmentation of anatomical structures: significantly improved efficiency and reliability. Neuroimage 31, 1116-1128 (2006)

38. Zhang, Y.J.: A survey on evaluation methods for image segmentation. Pattern Recognit. 29, 1335-1346 (1996)

39. Zheng, Y., Comaniciu, D.: Marginal Space Learning for Medical Image Analysis: Efficient Detection and Segmentation of Anatomical Structures. Springer, New York (2014)

40. Zhu, L., Kolesov, I., Gao, Y., Kikinis, R., Tannenbaum, A.: An effective interactive medical image segmentation method using fast growcut. In: Workshop on Interactive Medical Image Computing (MICCAI), pp. 1-9 (2014)

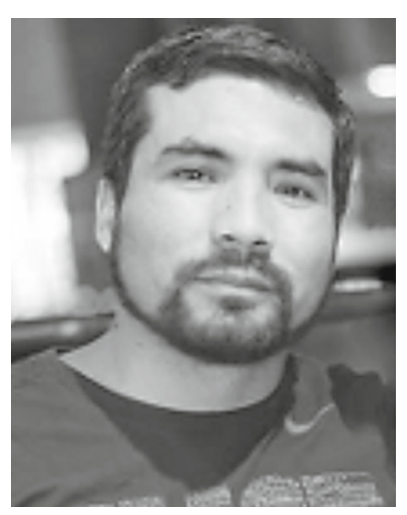

Oscar Cuadros Linares received his BS degree in Informatics Engineering at the Catholic San Pablo University, Arequipa, Peru in 2009. He received his MSc degree in Computer Science at the University of Sao Paulo, Brazil, in 2013. In 2017 obtained his Ph.D. degree in Computer Science at the University of Sao Paulo, Brazil. His areas of interest are image processing, graph theory and complex networks.

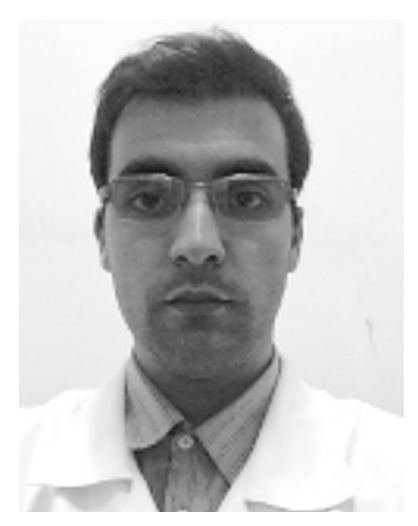

Jonas Bianchi received his DDS degree in 2014 and in 2017 obtain his MSD degree with emphasis in Orthodontics at the Sao Paulo State University (UNESP), School of Dentistry, Araraquara, Brazil. $\mathrm{He}$ is currently attending the DDSc degree in the same institution. His research interest includes techniques for CBCT imaging applied in medical and dental fields, evaluation of surgical and orthodontic treatments, mainly focused in effects of orthognathic surgery and pathology involving the temporomandibular joint.

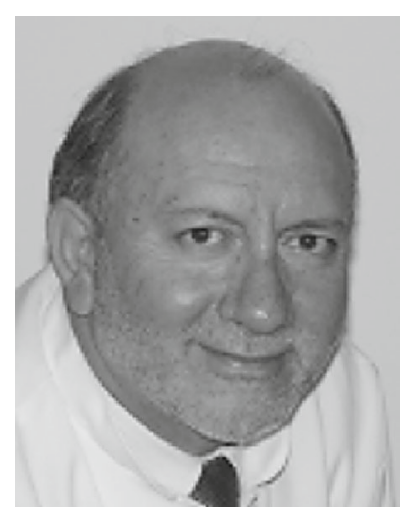

Dirceu Raveli received DDS degree from UNICAMP-Faculty of Dentistry, Piracicaba, SP, in 1975. $\mathrm{He}$ received his MSc degree in Pediatrics Dentistry from UNESP -Faculty of Dentistry, Araraquara, SP, in 1982. He received his $\mathrm{PhD}$ degree from UNESPFaculty of Dentistry, Araraquara, SP, in 1985. He became an Associate Professor in Orthodontics at UNESP-Faculty of Dentistry, Araraquara, SP, in 1998. From 1982 to the moment, he is a professor in Faculty of Dentistry, Unesp, Araraquara, SP, at the Clinical and Research Program with the interest including growth and development of face and effects of orthopedics appliances. 


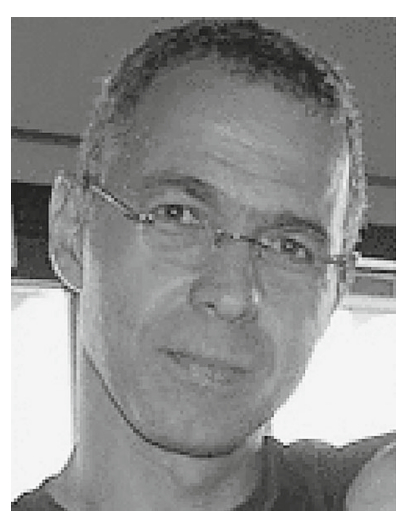

João Batista Neto is a computer scientist. He received the $\mathrm{PhD}$ degree from Imperial College, London, in 1996. He is currently working at University of Sao Paulo, and his major research activities concern image processing and image analysis.

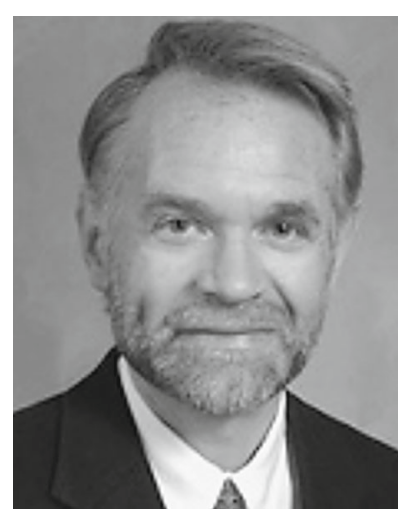

Bernd Hamann studied computer science and mathematics at the Technical University of Braunschweig, Germany, and computer science at Arizona State University, USA. He is a professor at the University of California, Davis. His main teaching and research interests are data analysis and visualization, geometric design and modeling, image processing and computer graphics. 